\title{
Artificial Neural Networks for cosmic gamma-ray propagation in the Universe
}

\author{
K. K. Singh ${ }^{\mathrm{a}, *}$, V. K. Dhar ${ }^{\mathrm{a}}$, P. J. Meintjes ${ }^{\mathrm{b}}$ \\ ${ }^{a}$ Astrophysical Sciences Division, Bhabha Atomic Research Centre, Mumbai- 400 085, \\ India \\ ${ }^{\mathrm{b}}$ Physics Department, University of the Free State, Bloemfontein - 9300, South Africa
}

\begin{abstract}
We explore the potential of an artificial neural network (ANN) based method intelligence to probe the propagation of cosmic $\gamma$-ray photons in the extragalactic Universe. The journey of $\gamma$-rays emitted from a distant source like blazar to the observer at the Earth is impeded by the absorption through the interaction with the extragalactic background light (EBL), leading to an electron-positron pair production. This process dominates for gamma ray photons with energy above $10 \mathrm{GeV}$ propagating over the cosmological distances. The effect of $\gamma$ ray attenuation is characterized by a physical quantity called optical depth, which strongly depends on the $\gamma$-ray photon energy, redshift of the source, and density of the EBL photons. We estimate the optical depth values for $\gamma$-ray energies above $10 \mathrm{GeV}$ emitted from the sources at redshifts in the range 0.01 to 1 using three different and most promising EBL models. These optical depth estimates are randomly divided into two data sets for training and testing of the ANN using energy, redshift as inputs and optical depth as output. The optimization of ANN-performance for each EBL model employs standard back-propagation (BP) and radial-basis function (RBF) algorithms. The performance of the ANN model using the RBF is found to be superior to the BP method. In particular, the RBF-ANN with 40 neurons in the hidden layer corresponding to the EBL model proposed by Finke et al. (2010) shows the best performance for the propagation of $\gamma$-rays in the Universe.
\end{abstract}

Key words: gamma-rays; extragalactic background light; photon-photon interaction; artificial neural network

\footnotetext{
* Corresponding author.

Email address: kksastro@barc.gov. in (K. K. Singh).
} 


\section{Introduction}

In the last two decades, the machine learning approaches using artificial neural networks (ANNs) have received a significant attention for solving a variety of problems in astrophysics and cosmology [1,2,3,4,5,6,7]. Machine learning methods refer to the group of algorithms which can solve new problems on the basis of learning from the existing examples. They are generally divided into two main categories namely the supervised and unsupervised learning. In supervised learning, the algorithms learn from a correctly labeled data through the mapping of a set of features in the input data set to the output variable and construct a model from the input data [8]. The unsupervised algorithms learn from the complex relationships existing in the unlabeled data set comprising measured features and have several external free parameters [9]. The ANNs are introduced as a non-linear mathematical technique for data prediction on the basis of input training data. The ANN can solve problems which are based on non-linear correlations and pattern recognition. Some of the practically solvable problems using the ANN include object localization, forecasting, approximation, data compression, clustering, astrophysical classification, adaptive optics in the astronomical observations, parameter extraction for the $21 \mathrm{~cm}$ Global signal, search for gravitational wave signals, modelling the dusty Universe etc. A hybrid model based on ANN has been proposed to predict spectral energy distributions of galaxies at wavelengths ranging from the optical to the radio [10]. Deep learning models, which make use of long interconnected layers of ANN with powerful learning algorithms, have also emerged as the state of the art techniques to achieve outstanding results in different fields from computer vision, image recognition to the astrophysics and fundamental research $[11,12,13,14,15,16]$.

The Universe comprises very powerful celestial $\gamma$-ray sources which emit a strong beam of photons with energy above few $\mathrm{MeV}$. The $\gamma$ ray photons in the $\mathrm{MeV}-\mathrm{GeV}$ energy range are characterized as high energy (HE) and those with energy above $100 \mathrm{GeV}$ are referred to as very high energy (VHE) in modern astronomy [17]. The observations of celestial HE and VHE sources using the space and groundbased telescopes strongly depend on the nature of $\gamma$ ray photon propagation over cosmological distances in the Universe. The propagation of $\gamma$ ray photons emitted from the sources at cosmological redshifts is strongly affected by the presence of the extragalactic background light (EBL) $[18,19]$ and the extragalactic magnetic field [20]. Due to the effect of EBL, the observed $\gamma$-ray spectra of the extragalactic sources differ significantly from the intrinsic spectra emitted at the source. This is attributed to the absorption of $\gamma$ ray photons by the low energy EBL photons via $\gamma-\gamma$ pair production $[21,22]$. However, oscillations between $\gamma$ ray photons and axion-like particles due to the extragalactic magnetic field lead to a reduction in the EBL absorption [20]. Therefore, the observed $\gamma$-ray spectra are the convolution of the photon-photon interactions and photon- axion-like particles oscillations. Both processes exhibit uncertainty due to a lack of precise measurement of the EBL spectral energy distribution, strength of the extragalactic magnetic field and existence 
of hypothetical axions beyond the standard model [23]. The EBL plays a dominant role in the propagation of $\gamma$ ray photons and limits the transparency of the Universe beyond a certain redshift [19]. It also introduces specific spectral signatures in the observed VHE spectra of the extragalactic sources. Therefore, the interpretation of $\gamma$-ray propagation in the extragalactic space is crucial to reconstruct the intrinsic VHE spectra of the sources which are indicative of the physical processes involved in the non-thermal emission. The propagation effects of the $\gamma$ ray photons also provide a way to indirectly probe the EBL which is an important cosmological quantity after the cosmic microwave background radiation and characterizes the star formation and evolution of galaxies in the Universe [19,24,25]. Direct measurements of the EBL are challenged by the strong foreground contamination due to the zodiacal light and diffuse Galactic light [18,26].

In this paper, we demonstrate that the ANN based module can be used as a tool to probe the propagation of $\gamma$ ray photons in the Universe. We use the opacity of the Universe caused by the EBL to $\gamma$-rays as a function of the observed photon energy $(E)$ and source redshift $(z)$ corresponding to three different popular EBL models for optimizing the performance of the ANN-module. We begin with a brief introduction to the EBL models in Section 2. In Section 3, we discuss the theory of $\gamma$-ray propagation over cosmological redshifts. The application of ANN in the $\gamma$-ray propagation is described in Section 4. Finally, we discuss and conclude the important findings of the study in Section 5.

\section{Extragalactic Background Light}

The EBL is the integrated diffuse radiation emitted from the stars and active galaxies through thermal and non-thermal processes and reprocessed by the interstellar dust over the history of the Universe [18]. Its spectral energy distribution ranges from the ultraviolet-optical (UV-Opt) to far-infrared (FIR) and exhibits two distinct humps. The first hump peaking at $\sim 1 \mathrm{eV}$ is characterized as the UV-Opt emission directly from the stars and the second one peaking at $\sim 0.01 \mathrm{eV}$ is ascribed as the IR emission due to the absorption and reprocessing of the stellar light by the dust present in the inter-galactic space. Therefore, the EBL spectrum carries important information about the star formation history and evolution of galaxies in the Universe. The density of the EBL photons is $\left.10^{-6}-10^{4}\right) \mathrm{ph} \mathrm{cm}^{-3} \mathrm{eV}^{-1}$ and their wavelength is redshifted due to the expansion of the Universe. The integrated EBL intensity represents a dominant component of the total energy budget of the Universe and the EBL contribution to the total energy density is $\sim 15 \%$ of that due to the cosmic microwave background radiation [27]. The spectrum of the EBL at the present epoch is not completely known due to the uncertainties in the relevant astrophysical processes like stellar formation, evolution, reprocessing of light by cosmic dust and other gravitational and nuclear processes contributing to the EBL. However, several models for the spectral energy distribution of the EBL over a 
wide wavelength range have been developed using different empirical and computational approaches like forward evolution, backward evolution and semi-analytical $[19,28]$. A brief description of the three widely accepted and used EBL models is given below.

\subsection{Franceschini et al. (2008): Model-A}

The EBL model proposed by Franceschini et al. (2008) is a backward evolution model based on the extrapolation of the low redshift galaxy data to higher redshifts under certain assumptions on the evolution of the galaxies in the Universe [29]. In this model, the EBL photon density and its evolution as a function of redshift are estimated from the fitting and interpolation of the multi-wavelength data available at UV-optical and IR wavelengths from various ground and space-based astronomical observations. Additional constraints on the spectral energy distribution of the EBL are obtained from the dedicated direct measurements at known wavelengths. Recently, the model has been reviewed on the basis of new optical and IR deep survey observations [30]. However, no significant modification on the EBL photon density is obtained at UV-optical and IR wavelengths. We have used the original EBL determinations reported in [29] in the present study and refer to this as Model-A.

\subsection{Finke et al. (2010): Model-B}

In this model, the EBL intensity at UV-optical and IR wavelengths is estimated by assuming the main and post-main sequence stars as blackbodies and reprocessing of starlight by cosmic dust respectively [31]. This model is not based on the galaxy data and the evolution of galaxies over cosmological redshifts is inferred from the observed star formation rate density and initial mass function. The reprocessing of starlight absorbed by dust is modelled as IR emissions by three blackbodies representing warm (large), hot (small) dust grains and polycyclic aromatic hydrocarbons. The spectral energy distribution of the EBL estimated in [31] is close to the lower limits from the galaxy counts. This model provides the most accurate estimates for the density of the EBL photons at low redshifts and short wavelengths. We refer to this EBL spectral energy distribution as Model-B in the present study.

\subsection{Domínguez et al. (2011): Model-C}

Domínguez et al. (2011) have derived the evolving spectral energy distribution of the EBL on the basis of observations only [32]. The novel method employed to estimate the EBL spectrum uses the observed evolution of the rest-frame galaxy luminosity function in K-band up to redshift $z \sim 4$ and changing fractions of the 


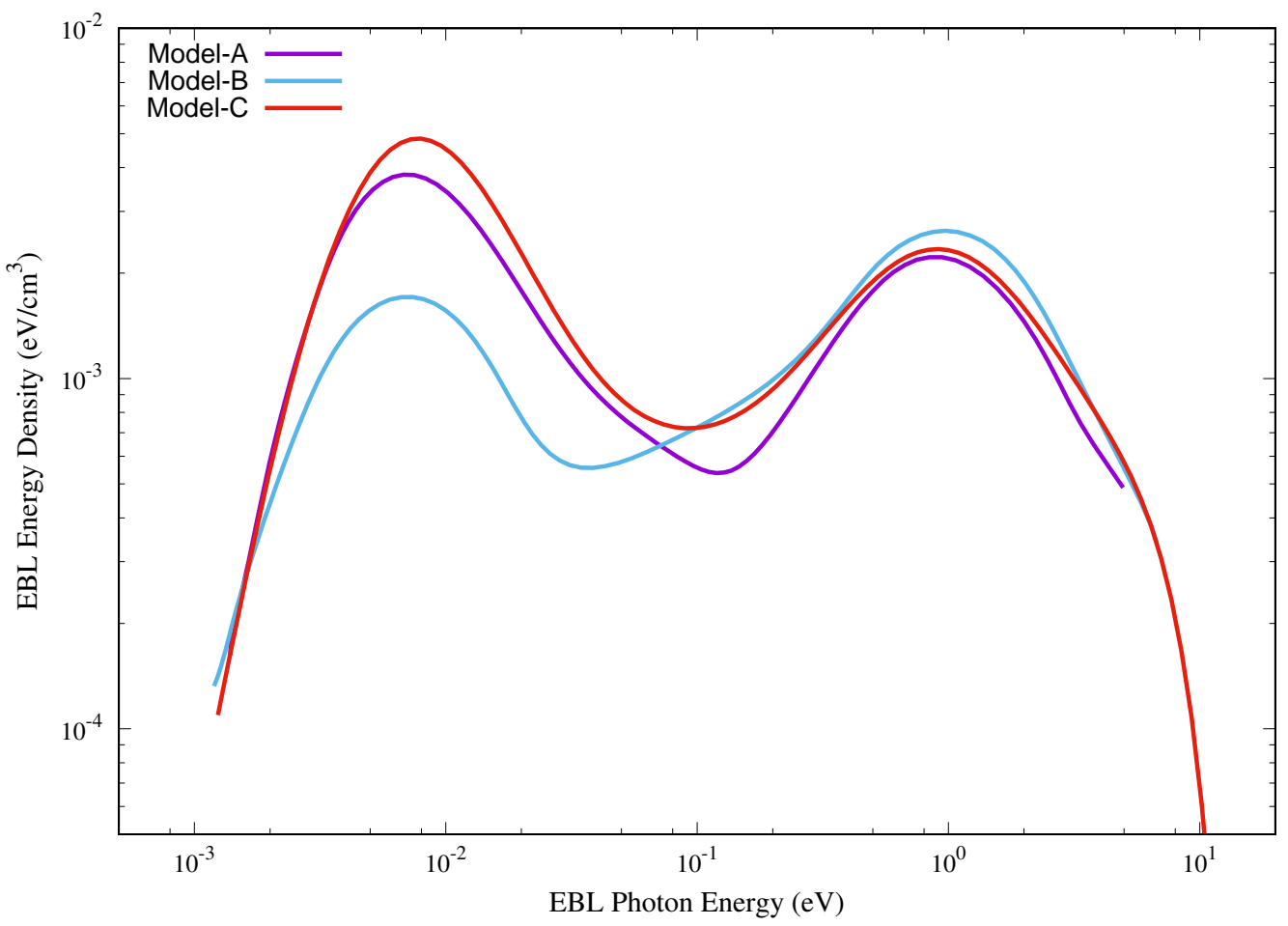

Fig. 1. Spectral energy distributions of the EBL proposed by three different models: Model-A [29], Model-B [31], and Model-C [32].

quiescent galaxies to fit the observed multi-wavelength templates. The evolving contribution to the bolometric EBL from UV to IR due to different populations of galaxies is calculated from the extrapolation of the galaxy spectral energy distribution type fractions. The modelling of EBL in [32] has two major shortcomings namely extrapolation of the galaxy fractions at higher redshifts and colour dependent selection effects. The EBL spectrum obtained by this model is consistent with the majority of the data from galaxy counts. We define this as Model-C in the present study.

The energy density of EBL photons predicted by the above models is shown in Figure 1. These models are generally used in the study of the opacity of the Universe to VHE $\gamma$ ray photons. Therefore, we have employed these three models in the present work to explore the potential of ANN in probing the $\gamma$-ray propagation in the Universe.

\section{$3 \gamma$-ray Propagation}

The propagation of $\gamma$ ray photons over cosmological distances is hindered due to the presence of EBL via $\gamma-\gamma$ absorption. The $\gamma$ ray photons emitted from a distant cosmic source and propagating in the intergalactic space can be absorbed by the 
EBL photons through the Breit-Wheeler process which is expressed as [33]

$$
\gamma+\gamma_{E B L} \rightarrow e^{-}+e^{+}
$$

This process is kinematically allowed when the following threshold condition is satisfied [34]

$$
E \epsilon=\frac{2 m_{e}^{2} c^{4}}{(1+z)^{2}(1-\cos \theta)}
$$

where $E$ and $\epsilon$ are the energies of $\gamma$-ray and EBL photons measured at the present epoch respectively, $\theta$ is the angle between the directions of the motion of two photons and $m_{e} c^{2}$ is the rest mass energy of the electron. The $(1+z)^{2}$ factor takes into account the change in energy of photons due to the cosmic expansion of the Universe. For an isotropic distribution of the EBL photons, the minimum energy of the background photons for the maximum $\gamma-\gamma$ absorption is given by [34]

$$
\epsilon \approx\left(\frac{900}{(1+z)^{2} E(G e V)}\right) \mathrm{eV}
$$

This suggests that for VHE $\gamma$-ray photons with energy in the range $100 \mathrm{GeV} \leq E \leq$ $20 \mathrm{TeV}$ emitted from the sources at redshift $z \sim 1$, the energy of the background photons for the absorption via pair production should be $2.2 \mathrm{eV} \leq \epsilon \leq 0.011 \mathrm{eV}$. Therefore, the EBL photons play a dominant role in the absorption of VHE $\gamma$-ray photons propagating over cosmological distances $(z>0)$ in the Universe. This process leads to an energy $(E)$ and redshift $(z)$ dependent depletion of the VHE $\gamma$-ray flux from a distant cosmic source and therefore limits the transparency of the Universe to $\gamma$-rays. The survival probability (i.e probability of escaping from the EBL absorption) of VHE $\gamma$-ray photons is estimated as

$$
P_{\gamma \rightarrow \gamma}=e^{-\tau(E, z)}
$$

where $\tau$ is referred to as the optical-depth, which characterizes the opacity of the Universe and strongly depends on $E$ and $z$ for a given spectral energy distribution of the EBL photons. By definition, $\tau(E, z)=1$, is termed as " $\gamma$-ray horizon" and $\tau(E, z)<1$ or $\tau(E, z)>1$ corresponds to transparent or opaque Universe respectively. The optical depth $\tau(E, z)$ for a given EBL model is computed from the following three fold integral $[25,34]$

$$
\tau\left(E, z_{s}\right)=\int_{0}^{z_{s}} \frac{d l}{d z} d z \int_{0}^{\pi}\left(\frac{1-\cos \theta}{2}\right) \sin \theta \mathrm{d} \theta \int_{\epsilon_{\min }}^{\infty} \mathrm{n}(\epsilon, \mathrm{z}) \sigma_{\gamma \gamma}(\mathrm{E}, \epsilon, \theta) \mathrm{d} \epsilon
$$

where $z_{s}$ is redshift of the $\gamma$-ray source, $\left(\frac{d l}{d z}\right)$ is the line element for the distance travelled by a VHE photon, $\mathrm{n}(\epsilon, z)$ is the number density of the EBL photons per unit energy, and $\sigma_{\gamma \gamma}(E, \epsilon, \theta)$ is the total scattering cross section for pair production. The lower limit of the integral over the energy of EBL photons $\left(\epsilon_{\min }\right)$ is given by the threshold condition for pair production from Equation 2. We have used the 
following set of parameters in the $\Lambda \mathrm{CDM}$ cosmology

$$
\frac{d l}{d z}=\frac{c}{H_{0}} \frac{1}{(1+z) \sqrt{\Omega_{\Lambda}+\Omega_{m}(1+z)^{3}}}
$$

with $\Omega_{m}=0.30, \Omega_{\Lambda}=0.70$ and $\mathrm{H}_{0}=70 \mathrm{~km} \mathrm{~s}^{-1} \mathrm{Mpc}^{-1}$ in the present work.

\section{Application of ANN to the gamma-ray propagation}

The ANN represents a simplified model of the human brain functioning and the nervous system. A human brain consists of more than $10^{10}$ neurons connected to each other with a complex topology and a simple ANN is described as a network of three inter-connecting layers namely input layer, hidden layer and output layer [35]. Each layer comprises of different number of neurons as basic building blocks and the number of neurons in the hidden layer determines the width of the ANN [36,37]. An ANN having several hidden layers due to the complexity of the problem is referred to as deep ANN. Each neuron in the input layer (first layer) is connected to one or more neurons in the hidden layer (intermediate layer). The individual connections between the neurons of the input and hidden layers are characterized by an appropriate weight and bias [38]. Each neuron in the hidden layer derives an activation through the linear combination of the input neurons from the input layer and the weights and biases associated with the respective connections. These activations are passed through linear or non-linear transformations to the output layer which performs a simple sum of all inputs from the hidden layer and predicts an output. This is referred to as the feed-forward process in the simple ANN model [39]. The width, deepness and activations of an ANN are decided in the beginning of the model, whereas weights and biases associated with the connections are free parameters and have to be optimized during the training process. To optimize the performance of an ANN model, a loss function or error function is defined. In the present work, we have used mean squared error (MSE) to measure the performance of the ANN model for the $\gamma$-ray propagation. It is defined as

$$
\operatorname{MSE}=\frac{1}{\mathrm{~N}} \Sigma_{\mathrm{i}=1}^{\mathrm{N}}\left(\mathrm{Y}_{\mathrm{d}}^{\mathrm{i}}-\mathrm{Y}_{\mathrm{p}}^{\mathrm{i}}\right)^{2}
$$

where $N$ is the total number of input samples, $Y_{d}^{i}$ and $Y_{p}^{i}$ are the desired (true) and ANN predicted outputs respectively for the $i^{\text {th }}$ sample. The mean squared error loss function gives outliers a larger weightage and needs to be minimized during the training for determining the free parameters like weights and biases in an excellent performing ANN. Different types of supervised and unsupervised algorithms are used to simulate the learning process of the ANN. In this study, we have employed two algorithms namely the standard Back-propagation (BP) and Radial basis function (RBF) to construct the neural network for probing the $\gamma$-ray propagation in the 


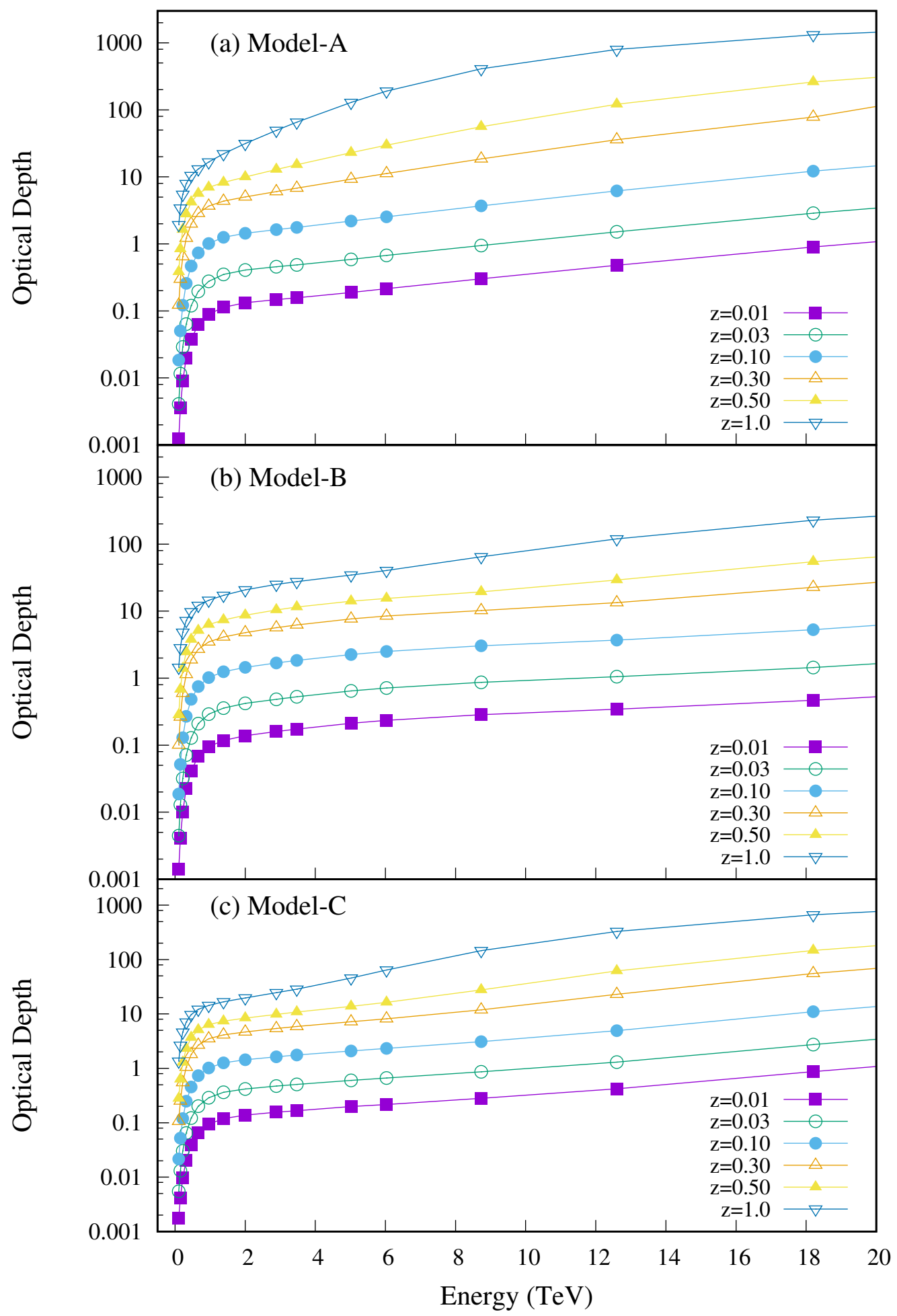

Fig. 2. Data set employed for training the ANN using three different EBL models. A similar data set is used for testing of the ANN. 
Universe. We have used an in-house developed software package BIKAS (Barc Iit KAnpur Simulator) for a major portion of the present work. Dhar et al. (2010) have reported a comparative study of a few popular ANN algorithms on the benchmark and function approximation problems using BIKAS package [40]. A brief and qualitative description of the $\mathrm{BP}$ and $\mathrm{RBF}$ algorithms used in the present study is given below.

\subsection{Back-propagation}

The back-propagation (BP) is a supervised learning algorithm which involves the gradient-descent paradigm to minimize the mean squared error [41]. In this algorithm, the weights are randomly initialized to small values to calculate the ANN output $\left(Y_{p}^{i}\right)$ and compare this with the desired output $\left(Y_{d}^{i}\right)$ value over every $i^{\text {th }}$ train-

ing input. If the values of $Y_{p}^{i}$ and $Y_{d}^{i}$ are not similar, the error or difference between these two values is computed. The errors are propagated backward from the output layer to the input layer after every epoch and the weights are adjusted according to the Delta Rule for minimizing the error value. This employs the modification of weights along the most direct path in the weight space through the slope or gradient of the error function. The changes in weights towards the error minimization are proportional to the negative gradient of the error function [42]. This process repeatedly adjusts the weights in the ANN until the mean squared error at the network ouput is minimized. The gradient-descent based BP is a popular method for training of the ANN due to its simplicity and operational ease. However, the selection of hidden layers is critical in the design of a BP-based neural network since there is no analytical approach for optimizing the deepness of an ANN. This is generally done through the proper training of the ANN on various permutations and selection of the minimum number of layers or neurons which can yield the lowest value of the mean squared error. In addition to this, the training of the BP-based ANN is quite time consuming and exhibits the local minima entrapment problem.

\subsection{Radial Basis Function}

The radial basis function ( $\mathrm{RBF}$ ) provides an efficient and attractive alternative to BP method for the training of the ANN $[43,44]$. The RBF-based ANN has a capability of universal function approximation and regularization. Unlike BP, RBF approach constructs approximation models assuming linear terms of the parameters or weights for any continuous function [45]. The training of the RBF-based ANN is faster than that of the BP since it is constrained to have just two layers of weigths which are sequentially fixed. The whole structure of the network is fixed only through the determination of the hidden layer and the weights associated with the connections between the hidden layer and ouput layer. The weights associated 
Table 1

Summary of the mean squared error for optimization of the number of neurons using the BP algorithm for Model-A.

\begin{tabular}{lccc}
\hline Number of Neurons & MSE & $\sigma$ (Training) & $\sigma$ (Testing) \\
\hline 5 & 3560.81 & 512.62 & 788.32 \\
10 & 681.09 & 213.75 & 311.81 \\
15 & 144.46 & 97.35 & 128.52 \\
20 & 47.49 & 40.42 & 78.51 \\
25 & 17.49 & 26.21 & 42.96 \\
30 & 8.02 & 20.51 & 31.91 \\
$\mathbf{3 5}$ & $\mathbf{2 . 1 4}$ & $\mathbf{1 3 . 2 1}$ & $\mathbf{1 8 . 3 6}$ \\
40 & 0.34 & 7.82 & 46.53 \\
\hline
\end{tabular}

with the connections between the input layer and hidden layer are decided after fixing the hidden layer. The weights of the hidden layer neurons are assigned in such a way that the network produces the maximum output for inputs equal to their weights and are not initialized randomly unlike BP. The RBF algorithm derives its working methodology from the famous Cover's Theorem, which states that a linearly non-separable data set in a low dimensional space can be transformed into a linearly separable data set in a high dimensional space through some non-linear transformation. The most commonly used activation function in the RBF-based ANN is a Gaussian function. The RBF algorithms do not exhibit any of the BP training anomalies like the problem of local minima.

The main objective of this work is to explore the potential of the ANN for probing the propagation of $\gamma$-ray photons over cosmological distances in the Universe. As discussed in Section 3, the $\gamma$-ray propagation is characterized by the optical depth $(\tau)$ which is a function of the redshift $(z)$ and energy of the photon $(E)$ for a given EBL model. We have estimated $\tau$ values for the $\gamma$-ray photons in the energy range $E=10 \mathrm{GeV}-20 \mathrm{TeV}$ at redshifts $z=0.01,0.03,0.10,0.30,0.50,1.0$ corresponding to the three different and most promising EBL models designated as Model-A, Model-B, and Model-C. We randomly split this data set into Training and Testing samples for optimization of the ANN performance using BP and RBF algorithms. The data samples used for training of the ANN for three EBL models are depicted in Figure 2. A similar data set is generated for the purpose of testing the ANN. 
Table 2

Same as Table 1 for Model-B.

\begin{tabular}{lccc}
\hline Number of Neurons & MSE & $\sigma$ (Training) & $\sigma$ (Testing) \\
\hline 5 & 212.33 & 79.22 & 92.15 \\
10 & 63.026 & 54.99 & 79.43 \\
15 & 14.024 & 36.37 & 66.79 \\
20 & 3.38 & 27.96 & 42.12 \\
25 & 0.63 & 22.10 & 31.47 \\
30 & 0.28 & 18.49 & 26.51 \\
$\mathbf{3 5}$ & $\mathbf{0 . 0 9}$ & $\mathbf{8 . 9 2}$ & $\mathbf{1 0 . 7 8}$ \\
40 & 0.02 & 6.41 & 12.47 \\
\hline
\end{tabular}

Table 3

Same as Table 1 for Model-C.

\begin{tabular}{lccc}
\hline Number of Neurons & MSE & $\sigma$ (Training) & $\sigma$ (Testing) \\
\hline 5 & 1366.95 & 460.51 & 635.92 \\
10 & 164.13 & 212.82 & 501.32 \\
15 & 28.20 & 102.37 & 332.46 \\
20 & 8.19 & 61.92 & 212.31 \\
25 & 1.22 & 41.36 & 101.32 \\
30 & 0.32 & 27.21 & 47.21 \\
35 & 0.08 & 19.22 & 27.92 \\
$\mathbf{4 0}$ & $\mathbf{0 . 1 3 2}$ & $\mathbf{7 . 2 1}$ & $\mathbf{1 3 . 2 9}$ \\
\hline
\end{tabular}

\subsection{Training and Testing of the ANN}

The most important requirement for the training of an ANN module is that a well trained ANN should work satisfactorily on a new input data set (testing) which was not used in the training process. Therefore, a high quality training data is crucial for the optimization of an ANN module for further applications. In this work, the training data set consist of $E$ and $z$ as inputs and $\tau$ as the desired ouput for three different EBL models (Figure 2). The first step in the training stage involves determining the optimized number of neurons to be employed in the ANN module. We have followed a trial and error method for optimizing the number of neurons during 
Table 4

Summary of the mean squared error for optimization of the number of neurons using the RBF algorithm for Model-A.

\begin{tabular}{lccc}
\hline Number of Neurons & MSE & $\sigma$ (Training) & $\sigma$ (Testing) \\
\hline 5 & 113.56 & 368.51 & 507.48 \\
10 & 24.19 & 55.74 & 80.30 \\
15 & 1.16 & 23.39 & 42.13 \\
20 & 0.76 & 17.54 & 31.12 \\
25 & 0.46 & 9.15 & 30.36 \\
30 & 0.24 & 7.92 & 11.21 \\
$\mathbf{3 5}$ & $\mathbf{0 . 2 2}$ & $\mathbf{5 . 2 1}$ & $\mathbf{1 1 . 8 1}$ \\
40 & 0.18 & 3.41 & 15.42 \\
\hline
\end{tabular}

Table 5

Same as Table 4 for Model-B.

\begin{tabular}{lccc}
\hline Number of Neurons & MSE & $\sigma$ (Training) & $\sigma$ (Testing) \\
\hline 5 & 122.32 & 66.61 & 78.36 \\
10 & 37.30 & 38.42 & 67.11 \\
15 & 9.02 & 24.62 & 42.54 \\
20 & 1.27 & 19.96 & 32.93 \\
25 & 0.49 & 16.39 & 26.43 \\
30 & 0.22 & 12.23 & 21.51 \\
35 & 0.14 & 9.71 & 15.02 \\
$\mathbf{4 0}$ & $\mathbf{0 . 0 7}$ & $\mathbf{7 . 0 9}$ & $\mathbf{8 . 4 7}$ \\
45 & 0.05 & 6.32 & 14.87 \\
\hline
\end{tabular}

the training process [46]. The details of the optimization process for the number of neurons by minimizing the mean squared error using the BP and RBF algorithms are given in Table (1-3) and Table (4-6) respectively for three different EBL models with 12000 iterations in each case.

We have also computed the standard deviation $(\sigma)$ of the errors between expected output and ANN predicted value for the training as well as testing data sets. It is important to mention here that the BP is a standard algorithm for minimization of the mean squared error in neural networks. Therefore, we have compared the per- 
Table 6

Same as Table 4 for Model-C.

\begin{tabular}{lccc}
\hline Number of Neurons & MSE & $\sigma$ (Training) & $\sigma($ Testing) \\
\hline 5 & 79.26 & 36.18 & 84.97 \\
10 & 29.81 & 21.16 & 67.14 \\
15 & 7.36 & 17.41 & 34.21 \\
20 & 2.31 & 13.51 & 21.14 \\
25 & 0.98 & 10.94 & 15.92 \\
$\mathbf{3 0}$ & $\mathbf{0 . 1 3}$ & $\mathbf{7 . 6 9}$ & $\mathbf{9 . 0 6}$ \\
35 & 0.02 & 3.24 & 11.18 \\
\hline
\end{tabular}

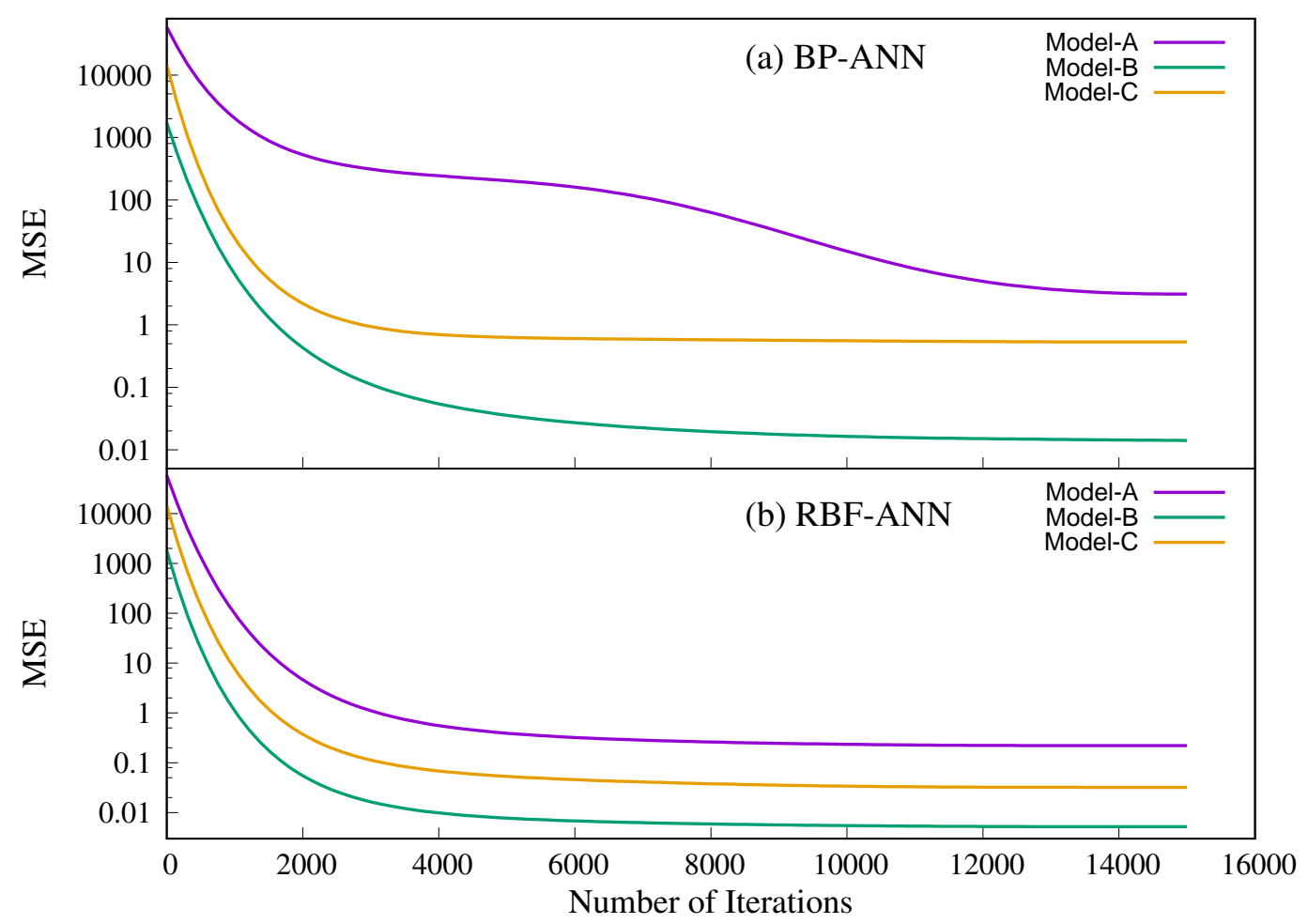

Fig. 3. Mean Squared Error during the training of ANN using two different algorithms (a) Back-propagation (BP) and (b) Radial basis function (RBF), corresponding to three EBL models: Model-A, Model-B, and Model-C.

formance of these two algorithms in the present work. The combinations of the optimized number of neurons corresponding to the minimum mean squared error values and a close matching between the $\sigma$-values for the training and testing data sets are shown as boldfaces in Tables (1-6). We note that a close matching between the $\sigma$-values occurs for 35 neurons (in one hidden layer) for Model-A and Model$\mathrm{B}$ and for 40 neurons for Model-C in the case of BP method in the present study 
(Table 1-3). Whereas, in the case of RBF algorithm, the corresponding number of neurons are 35, 40 and 30 for Model-A, Model-B and Model-C respectively (Table 4-6). These are referred to as optimum neurons and are further used to optimize the number of iterations, which is relatively an easier task. The resulting mean squared error as a function of the number of iterations for the BP and RBF algorithms is shown in Figure 3 (a) and (b) respectively. It is evident from the figure that the least mean squared error is observed for the Model-B after $\sim 10000$ iterations for BP as well as RBF methods. A relatively worst performance is obtained for the Model-A with both minimizing methods. The minimum values of the mean squared error associated with the Model-B are 0.0963 and 0.0780 for the BP and RBF methods respectively. This implies that the performance of the RBF algorithm is better than the conventional BP method after about 12000 iterations for the application to $\gamma$-ray propagation problem. Since the number of neurons in the hidden layer is crucial in the design of an ANN, we have further employed the Singular Value Decomposition (SVD) method to validate the optimum neurons $[6,47,48]$. We consider Model-B for the SVD method because the ANN based on this model shows the best performance as discussed above. We deliberately use larger number of neurons (60 in the present case) in the hidden layer to generate the weight matrix $(F)$ from the output of each node before subjecting them to the non-linear transformations. With a total of 102 training patterns and one hidden layer of 60 neurons, the order of matrix $F$ will be $102 \times 60$. The singular value decomposition of matrix $F$ is given by [49]

$$
F=U S V^{T}
$$

where $U$ and $V$ are the orthogonal matrices, and $S$ is a digonal matrix with 102 rows and 60 columns. The matrix $S$ contains singular values of the weight matrix $F$ on its diagonal. The dominance of the significant singular values of $F$ is determined by the percentage of energy explained $\left(P_{e x}\right)$ which is defined as [49]

$$
P_{e x}=\frac{\sum_{i=1}^{q} S_{i}^{2}}{\sum_{i=1}^{p} S_{i}^{2}} \times 100
$$

where $S_{1}, S_{2}, S_{3} \ldots . S_{p}$ are the singular values of $F$ in the descending order. $p$ and $q$ represent total and dominant number of singular values respectively. $P_{e x}$ as a function of number of nodes in the hidden layer is shown in Figure 4 for the RBF based ANN using Model-B. It is obvious from the figure that $99.99 \%$ energy is retained with 37 neurons as compared to 40 neurons estimated from the mean squared error minimization (Table 5). Therefore, 37-40 neurons in principle can be considered as optimal neurons in the present study.

In order to further monitor the performance of the ANN for the propagation of $\gamma$ ray photons, we randomly select different combinations of $E$ and $z$ to predict the optical depth values by the above trained ANN corresponding to three EBL models. We also compute the corresponding $\tau$ values using Equation 5 for Models-A, $\mathrm{B}$, and $\mathrm{C}$. A comparison of the ANN predicted optical depth values with the desired values (from Equation 5) for three EBL models is depicted in Figure 5(a-c). This 


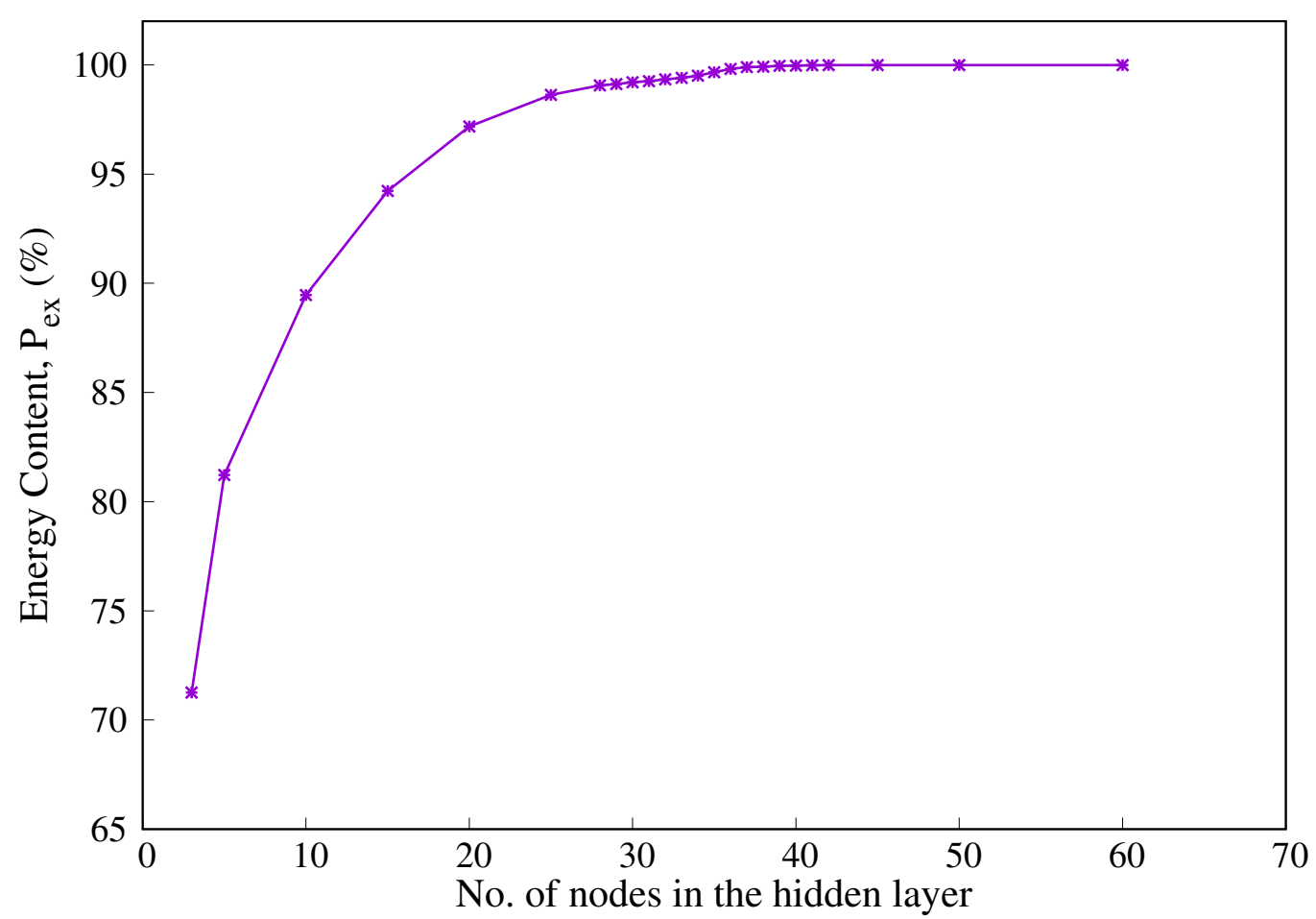

Fig. 4. Percentage of energy explained as a function of number of neurons in the hidden layer for the RBF-ANN corresponding to the Model-B of the EBL.

forms independent data sets (i.e. unseen during training and testing procedures) to measure the robustness of the BP and RBF-based ANN designed for the propagation of $\gamma$-rays in the present work. We observe from the scatter plot in Figure 5(a-c) that the optical depth values predicted by the BP and RBF-based ANN show a better linear correlation with those expected from a given EBL density for Model-B (Figure 5b), whereas a large deviation is found for Models-A and C (Figure 5a \& c). It is evident from Figure 3 and Figure 5 that the BP-algorithm does not converge in general and especially for Model-C (Figure $3 \mathrm{a}$ and Figure 5c). This can be attributed to the fact that the BP-algorithm is based on the gradient-descent method, which is known to suffer from the problems of getting stuck in the local minima and slow convergence. On the other hand, convergence of global minima is more probable in the second order methods like RBF and therefore these algorithms are inherently better suited for the problems with non-linearity. Also, the performance of all ANNs is worse at low opacity values. At lower opacity values, change in the mean squared error is small despite large percentage error. This can be the result of the mean squared error definition implemented in the present study. Since the mean squared error values are fed back to optimize the weights, the contribution of smaller mean squared error values is less in modifying the weights and hence the training is restricted. To quantify the strength of the linear correlation, we have also computed the Pearson coefficient using the ANN predicted and desired optical depth values for Model-B. We obtain Pearson coefficient values of $\sim 0.48$ and $\sim 0.93$ for the BP and RBF methods respectively. This indicates that the $\tau(E, z)$ val- 
ues predicted by the RBF-based ANN show a strong and positive correlation with those expected from the Model-B. Therefore, the performance of the RBF-based ANN with 40 optimum neurons for the propagation of $\gamma$-rays over cosmological distances in the framework of Model-B is found to be better than the BP-based ANN with 35 neurons in the present work. The number of neurons is an important consideration in the design and application of ANN to any problem at hand. If the number of neurons employed in the ANN is less or more than the optimal neurons, the network is accordingly said to be under or over trained. This can result in over fitting or remembering the training data rather than its generalization [7].

\section{Discussions and Conclusions}

We have used optical depth estimates from the three different EBL models for a comparative study of the performance of the BP and RBF based ANN in the propagation of $\gamma$ ray photons over cosmological distances in the Universe. A properly designed ANN predicts an output corresponding to a set of inputs after a proper training by a large number of a priori known set of inputs and their desired outputs. The propagation of $\gamma$ ray photons with an energy over $10 \mathrm{GeV}$ is strongly affected by the large uncertainties in the density of ultraviolet, optical and infrared components of the EBL photons. The present study suggests that an RBF-based ANN outperforms the conventional BP method for probing the opacity of the Universe to the $\gamma$-rays emitted from a source in the redshift range $z=0.01-1$. Among the three most promising EBL models considered in this study, we find that the overall performance of the RBF-based ANN module is in better agreement with the EBL model proposed by Finke et al. (2010). Recently, the optical depth estimates from a model-independent approach based on the GeV-TeV observations of blazars at various redshifts also suggest that the models proposed by Finke et al. (2010) and Domínguez et al. (2011) give a relatively better description of the spectral energy distribution of the EBL than many other models in the literature [50]. The combined observations of blazars from the Fermi-Large Area Telescope (LAT) and the ground-based Cherenkov telescopes have provided a unique tool to probe and constrain the density of EBL photons $[51,52,53]$. The role of the spectral energy distribution of EBL is extremely important in cosmology to probe the galaxy formation and evolution throughout the history of the Universe. The evolution of EBL has been recently reconstructed by using the Fermi-LAT observations of more than 700 active galaxies in the redshift range $z=0.03-3$ [54,55]. The star formation history determined from the Fermi-LAT measurements is in good agreement with the independent measurements from galaxy surveys with a peak at $z \sim 2$. A comparison of the cosmic $\gamma$-ray horizon $(\tau(E, z)=1)$ from the Fermi-LAT observations up to $z=1$ with the predictions from Models-A, B \& $\mathrm{C}$ and by the RBF-ANN corresponding to Model-B is shown in Figure 6. The Fermi-LAT measurements provide $\gamma$-ray horizon up to $1 \mathrm{TeV}$ only due to its observational energy 


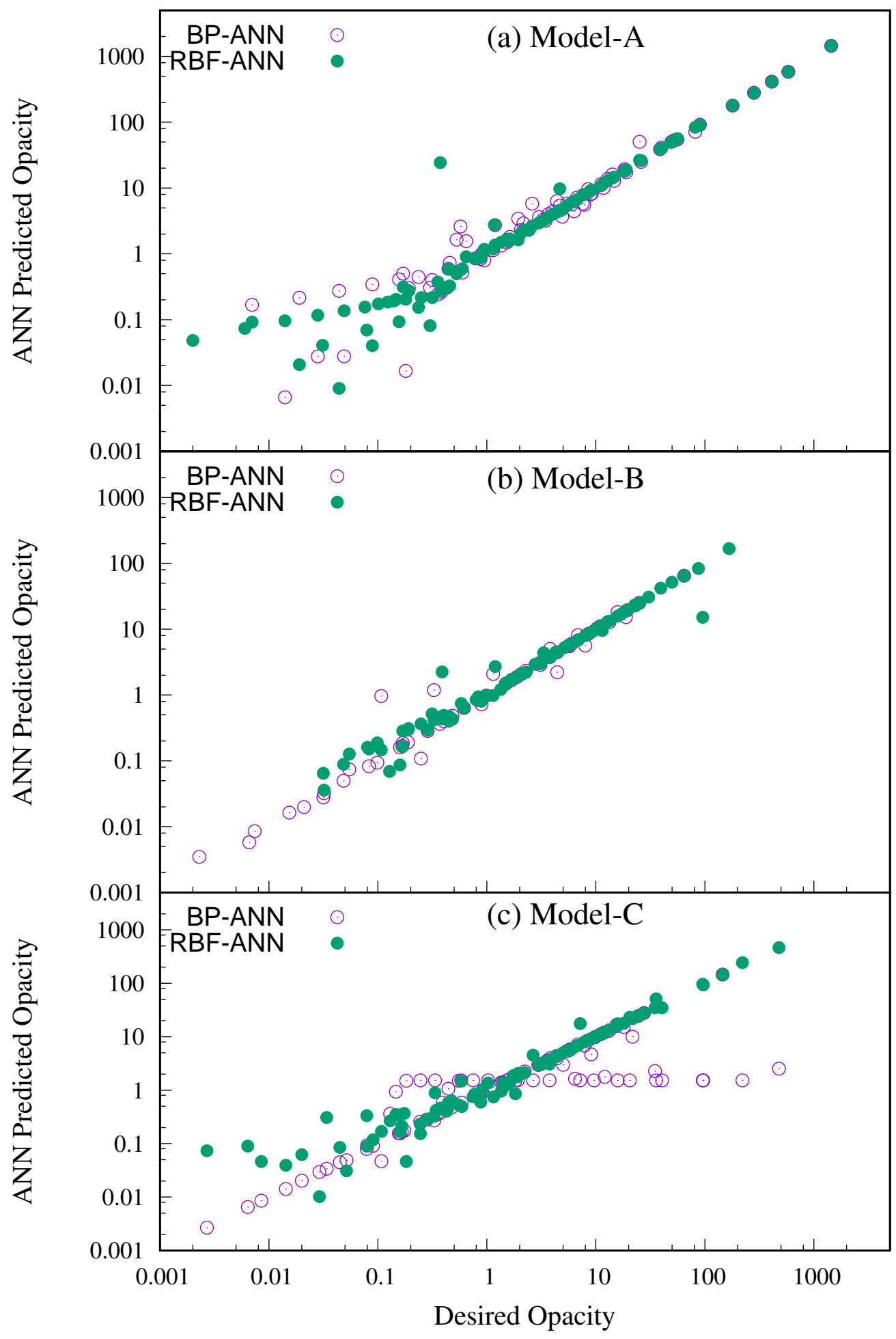

Fig. 5. Comparison of the optical depth values expected from three different EBL models with the corresponding values predicted by the BP and RBF-based ANN. 
range of $0.1 \mathrm{GeV}$ to less than $2 \mathrm{TeV}$. In this energy range, the cosmic $\gamma$-ray horizon predicted by the RBF-ANN corresponding to Model-B is broadly consistent with the Fermi-LAT measurements. Future $\gamma$-ray observations with the Cherenkov Telescope Array (CTA) over a wide energy range will be extremely helpful in probing the transparency of the Universe to higher redshifts and subsequently the spectral energy distribution of the EBL photons. This will enable a better understanding of the $\gamma$-ray propagation problem in cosmology.

In summary, the present study suggests that the RBF-based ANN module having 40 neurons in the hidden layer can describe the propagation of $\gamma$-rays in the Universe in a better agreement with the EBL model proposed by Finke et al. (2010). To the best of our knowledge, the application of RBF or BP-based ANN methodology for exploring the $\gamma$-ray propagation over the cosmological distances is demonstrated for the first time in the present work. The RBF algorithm being a powerful method, is found to be suitable for recovering the cosmic optical depth to $\gamma$ ray photons due to interaction with the EBL photons. Apart from estimation of the optical depth for a given $\gamma$-ray energy and source-redshift, the RBF-based ANN designed in the present work can also be effectively employed to explore the compatibility of the various existing EBL models with the independent measurements from $\gamma$-ray observations. Out of three prominent EBL models used in this study, Finke et al. (2010) model yields a solid result, where RBF-based ANN reproduces the opacities over the full parameter space and also in good agreement with the Fermi-LAT measurements. Therefore, the RBF-based methodology can be potentially used as an additional probe for different EBL models as it involves both the direct EBL measurements and the indirect opacities predicted by the $\gamma$-ray observation. Thus, the new knowledge contributed by the present study lies in the fact that the RBFapproach is most suitable to demonstrate the potential of ANN for probing the transparency of Universe to high energy $\gamma$-rays in general. Almeida et al. have successfully developed a new ANN approach in generating accurate predictions of the spectral energy distribution of the EBL photons using semi-analytical galaxy formation models [10]. This hybrid model can produce mock catalogues of galaxies for the forthcoming surveys for the extragalactic background radiation.

The performance of ANN in the present work can be further improved by including other physical processes related to the $\gamma$-ray propagation like photon- axionlike particles oscillations and cascading due to the presence of the extragalactic magnetic field. In the photon- axion-like particles coupling, VHE $\gamma$ ray photons in a beam can partially convert to axion-like particles during their journey from the source to the Earth in the presence of a coherent extragalactic magnetic field oriented in the direction transverse to the photon propagation [20]. The axion-like particles travel unimpeded over cosmological distances due to the negligibly small scattering cross section like neutrinos and convert back to the original $\gamma$-ray photons in the Galactic (Milky Way) magnetic field before reaching the Earth [20]. This process, generally referred to as the photon-axion-like particles oscillation, may enhance the transparency of the Universe to VHE $\gamma$ rays. Consequently, the effec- 


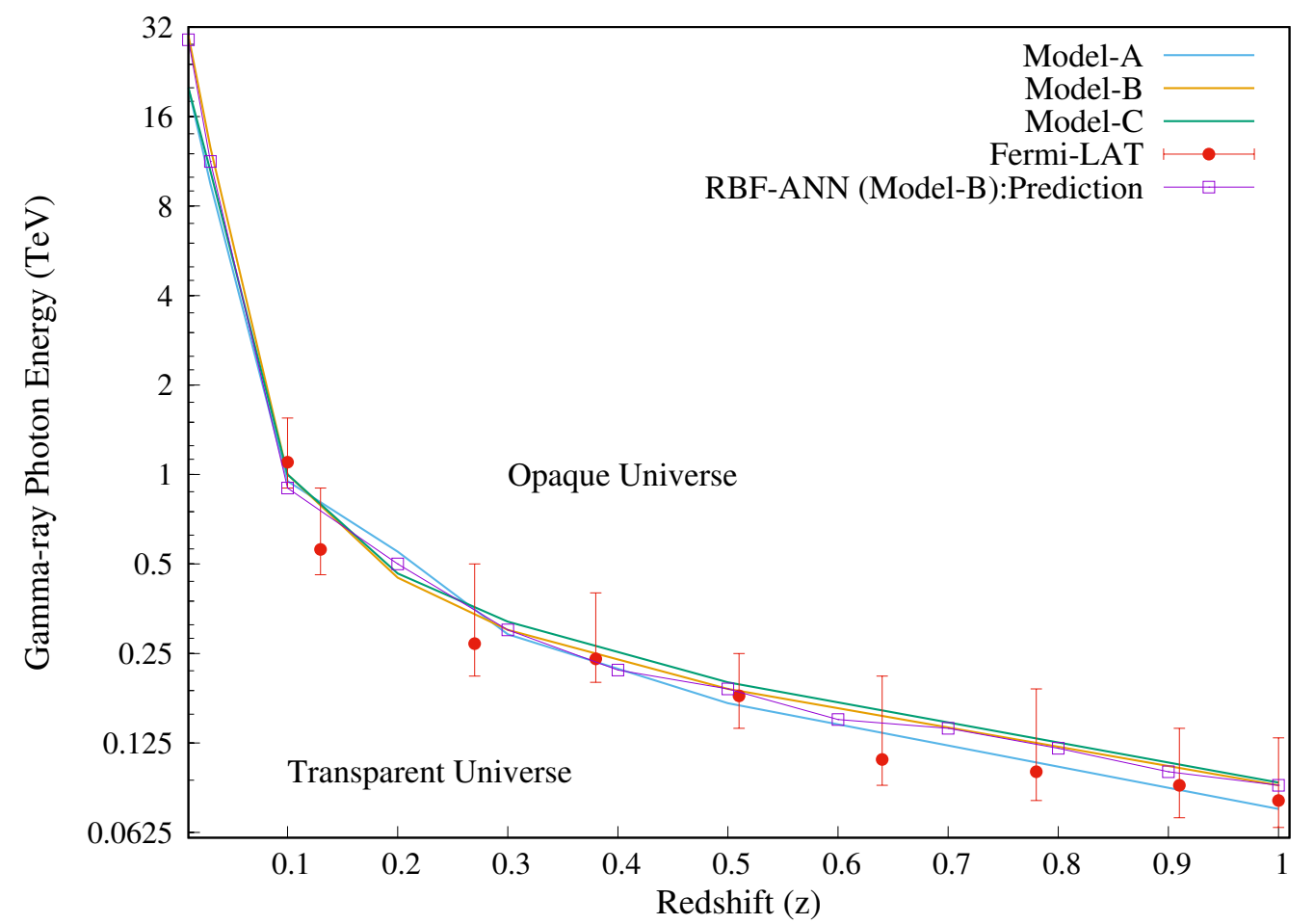

Fig. 6. Comparison of the cosmic gamma-ray horizon predicted by the RBF-based ANN designed using Model-B [31] and from the three different EBL models with the Fermi-LAT observations [54].

tive value of $\tau(E, z)$ will be reduced and the photon survival probability increases. Some observations with the modern ground and space-based instruments indicate that the Universe is more transparent to $\gamma$-rays than expected from the EBL absorption $[56,57,58]$. However, no direct experimental evidence regarding the existence of the axion-like particles has been obtained so far and only their parameter space (mass and coupling constant) is constrained at a given confidence level [59,60,61]. Also, the strength and coherence length of the extragalactic magnetic field are not clearly known so far and remain currently an active area of research.

\section{Acknowledgement}

Authors thank the anonyomus reviewer for her/his valuable suggestions to improve the contents of the manuscript. 


\section{References}

[1] Lahav O, Naim A, Sodré LJr, Storrie-Lombardi MC. Neural computation as a tool for galaxy classification: methods and examples. MNRAS 1996; 283: 207

[2] Andreon S, Gargiulo G, Longo G et al. Wide field imaging- I. Applications of neural networks to object detection and star/galaxy classification. MNRAS 2000; 319:700

[3] Cortiglioni F, Mähönen P, Hakala P et al. Automated Star-Galaxy Discrimination for Large Surveys. ApJ 2001; 556:937-943

[4] Ball NM, Loveday J, Fukugita NM et al. Galaxy types in the Sloan Digital Sky Survey using supervised artificial neural networks. MNRAS 2004; 348: 1038

[5] Teimoorinia H. Spectral Classification of Galaxies at $0.5 \leq \mathrm{z} \leq 1$ in the CDFS: The Artificial Neural Network Approach. AJ 2012; 144:172

[6] Dhar VK, Tickoo AK, Koul MK et al. Artificial Neural Network based $\gamma$-hadron segregation methodology for TACTIC telescope. NIMP A 2013; 708:56

[7] Singh KK, Dhar VK, Meintjes PJ. An artificial intelligence based approach for constraining the redshift of blazars using $\gamma$-ray observations. ExpA 2019; 48:297-311

[8] Reis I, Baron D, Shahaf S. Probabilistic Random Forest: A Machine Learning Algorithm for Noisy Data Sets. AJ 2019; 157:16

[9] Reis I, Poznanski D, Hall PB. Redshifted broad absorption line quasars found via machine-learned spectral similarity. MNRAS 2018; 480:3889-3897

[10] Almeida C, Baugh CM, Lacey CG et al. Modelling the dusty universe - I. Introducing the artificial neural network and first applications to luminosity and colour distributions. MNRAS 2010; 402:544

[11] Hinton GE, Osindero S, Teh, YB. A fast learning algorithm for deep belief nets. Neural computation $2006 ; 18: 1527$

[12] Yann L, Yoshua B, Hinton GE. Deep learning. Nature 2015; 521:436

[13] Silver D, Huang A, Maddison CJ. Mastering the game of Go with deep neural networks and tree search. Nature 2016; 529:484

[14] Baldi P, Sadowski P, Whiteson D. Searching for exotic particles in high-energy physics with deep learning. Nature Communications 2014; 5:4308

[15] Baldi P, Sadowski P, Whiteson D. Enhanced Higgs Boson to $\tau^{+} \tau^{-}$Search with Deep Learning. PRL 2015; 114:111801

[16] Baldi P, Bauer K, Eng C et al. Jet substructure classification in high-energy physics with deep neural networks. PRD 2016; 93:094034

[17] Aharonian F, Buckley J, Kifune T, Sinnis G. High energy astrophysics with groundbased gamma ray detectors. RPPh 2008; 71:096901 
[18] Hauser MG, Dwek E. The Cosmic Infrared Background: Measurements and Implications. ARA\&A 2001; 39:249-307

[19] Dwek E, Krennrich F. The extragalactic background light and the gamma-ray opacity of the universe. APh 2013; 43:112-133

[20] de Angelis A, Roncadelli M, Mansutti O. Evidence for a new light spin-zero boson from cosmological gamma-ray propagation. PhRvD 2007; 76:121301

[21] Stecker FW, de Jager OC, Salamon MH. TeV Gamma Rays from 3C 279: A Possible Probe of Origin and Intergalactic Infrared Radiation Fields. ApJL 1992; 390: L49

[22] Stecker FW, Malkan MA, Scully, ST. Intergalactic Photon Spectra from the Far-IR to the UV Lyman Limit for $0<\mathrm{z}<6$ and the Optical Depth of the Universe to High-Energy Gamma Rays. ApJ 2006; 648:774

[23] Peccei RD, Quinn HR. Constraints imposed by CP conservation in the presence of pseudoparticles. PhRvD 1977; 16:1791

[24] Costamante L. Gamma-Rays from Blazars and the Extragalactic Background Light. IJMPD 2013; 22:1330025-83

[25] Singh KK, Sahayanathan S, Tickoo AK, Bhatt N. Intrinsic VHE gamma-ray spectra of blazars as a probe for extragalactic background light. NewA 2014; 27: 34-40

[26] Hauser MG, Arendt RG, Kelsall T et al. The COBE Diffuse Infrared Background Experiment Search for the Cosmic Infrared Background. I. Limits and Detections. ApJ $1998 ; 508: 25-43$

[27] Berezinsky V, Gazizov A, Kalashev OV. ascade photons as test of protons in UHECR. APh 2016; 84:52-61

[28] Korochkin AA, Rubtsov GI. Constraining the star formation rate with the extragalactic background light. MNRAS 2018;481:557-565

[29] Franceschini A, Rodighiero G, Vaccari M. Extragalactic optical-infrared background radiation, its time evolution and the cosmic photon-photon opacity. A\&A 2008; 487:837852

[30] Franceschini A, Rodighiero G. The extragalactic background light revisited and the cosmic photon-photon opacity. A\&A 2017; 603: A34

[31] Finke JD, Razzaque S, Dermer CD. Modeling the Extragalactic Background Light from Stars and Dust. ApJ 2010; 712:238-249

[32] Domínguez A, Primack JR, Rosario DJ et al. Extragalactic background light inferred from AEGIS galaxy-SED-type fractions. MNRAS 2011; 410: 2556-2578

[33] Breit G, Wheeler JA. Collision of Two Light Quanta. PhRv 1934; 46:1087-1091

[34] Gould RJ, Schréder GP. Pair Production in Photon-Photon Collisions. PhRv 1967; 155:1404-1407

[35] Tagliaferri R, Longo G, Milano L et al. Neural Networks in Astronomy. Neural Networks 2003; 16:297-319 
[36] Fausett LV. Fundamentals of Neural Networks: Architectures, Algorithms And Applications. 1993

[37] Harvey RL. Neural Network Principles. 1994

[38] Rosenblatt F. The perceptron: a probabilistic model for information storage and organization in the brain. Psychological Review 1958; 65:386

[39] Terrence LF. Feedforward Neural Network Methodology. 2013

[40] Dhar VK, Tickoo AK, Koul R, Dubey BP. Comparative performance of some popular artificial neural network algorithms on benchmark and function approximation problems. Pramana 2010; 74:307-324

[41] Rumelhart DE, Hinton GE, Williams RJ. Learning representations by backpropagating errors. Nature 1986; 323:533-536

[42] McClelland JL, Rumelhart DE. Explorations in parallel distributed processing - A handbook of models, programs and exercises. 1988

[43] Broomhead DS, Lowe D. Multivariable Functional Interpolation and Adaptive Networks. Complex Systems 1988; 2:321-355

[44] Wu Y, Wang H, Zhang B, Du KL. Using Radial Basis Function Networks for Function Approximation and Classification. ISRN Applied Mathematics 2012; 1:324194

[45] Liao Y, Fang SC, Henry LW. Relaxed conditions for radial-basis function networks to be universal approximators. Neural Networks 2003; 16:1019-1028

[46] Haykin S. Neural Networks and Learning Machines. 1999

[47] Kanjilal PP, Dey PK, Banerjee DN. Reduced-size neural networks through singular value decomposition and subset selection. Electronics Letters 1993; 29:1516-1518

[48] Teoh EJ, Tan KC, Xiang C. Estimating the number of hidden neurons in a feedforward network using the singular value decomposition. IEEE TRANSACTIONS ON NEURAL NETWORKS 2006; 17:1623-1629

[49] Chakroborty S, Saha G. Feature selection using singular value decomposition and QR factorization with column pivoting for text-independent speaker identification. Speech Communication 2010; 52:693-709

[50] Singh KK, Meintjes PJ. Extragalactic background light models and GeV-TeV observation of blazars. NRIAG Journal of Astronomy and Geophysics 2020; 9:309-320

[51] Georganopoulos M, Finke JD, Reyes LC. A Method for Setting Upper Limits to the Extragalactic Background Light with Fermi-lat and TeV Observations of Blazars. ApJL 2010; 714:L157-L161

[52] Meyer M, Raue M, Mazin D, Horns D. Limits on the extragalactic background light in the Fermi era. A\&A 2012; 542:A59

[53] Desai A, Helgason K, Ajello M et al. A GeV-TeV Measurement of the Extragalactic Background Light. ApJL 2019; 874: L7 
[54] Abdollahi S, Ackermann M, Ajello $\mathrm{M}$ et al. A gamma-ray determination of the Universe's star formation history. Science 2018; 362:1031-1034

[55] Singh KK, Yadav KK, Meintjes PJ. Probing the evolution of the EBL photon density out to $z \sim 1$ via $\gamma$-ray propagation measurements with Fermi. Astrophys Space Sci. $2021 ; 366: 51$

[56] Albert J, Aliu E, Anderhub $\mathrm{H}$ et al. Very-High-Energy gamma rays from a Distant Quasar: How Transparent Is the Universe?. Science 2008; 320:1752

[57] Stecker FW, Scully ST. Is the Universe More Transparent to Gamma Rays Than Previously Thought?. ApJ 2009; 691: L91-L94

[58] Horns D, Meyer M. Indications for a pair-production anomaly from the propagation of VHE gamma-rays. JCAP 2012; 2012: 033

[59] de Angelis A, Galanti G, Roncadelli M. Relevance of axionlike particles for veryhigh-energy astrophysics. PhRvD 2011; 84:105030

[60] Ajello M, Albert A, Anderson B et al. Search for Spectral Irregularities due to PhotonAxionlike-Particle Oscillations with the Fermi Large Area Telescope. PhRvL 2016; 116:161101

[61] Long GB, Lin WP, Tam PH, Zhu WS. Testing the CIBER cosmic infrared background measurements and axionlike particles with observations of TeV blazars. PhRvD 2020; 101:063004 